\title{
Governance des Einkommensmix: Geringfügige Beschäftigung im ALG-II-Bezug
}

Ein Teil der Arbeitslosengeld (ALG)-II-Beziehenden arbeitet in einem Minijob. Diese Kombination von Erwerbseinkommen und Transferleistungen hat im Zeitverlauf kontinuierlich zugenommen. Auf Basis einer Analyse der Daten des Panels „Arbeitsmarkt und soziale Sicherung" können sowohl objektive Merkmale der Erwerbstätigkeit als auch subjektive Bewertungen der Lebenssituation dieser besonderen Beschäftigtengruppe dargestellt werden. Dabei zeigt sich, dass Minijobber, die zur Aufstockung ihres Niedrigeinkommens zugleich Leistungen aus dem ALG II beziehen, gegenüber anderen Minijobbern einer spezifischen Lohndiskriminierung unterliegen. Ferner deutet sich an, dass Minijobs eher als Aufstockung des Einkommens für dauerhaft Ausgeschlossene fungieren, denn als Brücke in reguläre Beschäftigung. ${ }^{\circ}$

IRENE DINGELDEY, PETER SOPP, ALEXANDRA WAGNER

\section{Problemhintergrund und Erkenntnisinteresse}

Minijobs sind per definitionem nicht Existenz sichernd, da die Einkommensgrenze von $400 €$ monatlich und der fehlende individuelle Zugang zu den Sozialversicherungen regulative Merkmale dieser spezifischen Beschäftigungsform darstellen. Minijobs werden deshalb tendenziell als Beschäftigungsverhältnisse zum „Zuverdienst“ angesehen. Sie werden jedoch auch mit (weiteren) Erwerbseinkommen und mit Transferleistungen kombiniert. Eine besondere Gruppe der Minijobberinnen und Minijobber sind die nach Sozialgesetzbuch Zweites Buch (SGB II) Leistungsberechtigten, die bei Bezug von Arbeitslosengeld II (ALG II) gleichzeitig Erwerbseinkommen aus einer Tätigkeit im Minijob erwirtschaften. Diese Gruppe wird im alltäglichen Sprachgebrauch und im Gegensatz zur offiziellen Terminologie der Bundesagentur für Arbeit (BA) häufig als Aufstocker bezeichnet. ${ }^{2}$

Der Umfang dieser Aufstocker ist in den letzten Jahren gestiegen. Seit Januar 2007 hat sich der Anteil der Minijobberinnen und Minijobber im SGB II nahezu kontinuierlich von unter $10 \%$ aller erwerbsfähigen Leistungsberechtigten (eLb) auf 15\% im April 2011 erhöht, sodass 698.000 der eLb geringfügig erwerbstätig waren (Bundesagentur für Arbeit 2011a, S. 22).

Wie sich die Governance des Einkommensmix im Rahmen verschiedener Regelungskontexte auf die Erwerbs- und Lebenssituation der Betroffenen auswirkt, ist weitgehend unbekannt. Frühere Untersuchungen beziehen sich entweder auf Regelungen und Entwicklung der Minijobs, ohne der spezifischen Situation von ALG-II-Beziehenden Rechnung zu tragen (Greve et al. 2007; Kirsch et al. 1999; Koch/Bäcker 2004; Winkel 2005) oder analysieren die Gruppe der erwerbstätigen Leistungsberechtigten insgesamt nach soziodemografischen Merkmalen, Haushaltskontext sowie Dauer des Leistungsbezuges, ohne auf die spezifische Situation der Minijobber einzugehen (Brenke 2008; Bruckmeier et al. 2007, 2008; Bundesagentur für Arbeit 2008, 2010a). Der Beitrag geht der Frage nach, ob die Governance des Einkommensmix für Minijobber im Leistungsbezug eine spezifische Erwerbs- und Lebenssituation generiert, die sich sowohl von anderen Minijobbern als auch von anderen nichterwerbstätigen Leistungsbeziehenden unterscheidet.

\footnotetext{
(1) Der vorliegende Artikel entstand im Rahmen des Projektes "Governance des Einkommensmix. Geringfügige Beschäftigung plus ALG-II-Bezug", das gemeinsam von der HansBöckler-Stiftung sowie der Arbeitnehmerkammer Bremen finanziert und 2010 am Institut Arbeit und Wirtschaft an der Universität Bremen durchgeführt wurde.
}

2 In der Leistungsstatistik der Bundesagentur für Arbeit werden Parallelbezieher von Arbeitslosengeld I und II als Aufstocker bzw. Aufstockerinnen bezeichnet. 
Dazu ist zunächst der spezifische Regelungskontext für die Kombination von ALG II und Minijobs zu betrachten (Abschnitt 2). Für die Charakterisierung erscheint zentral, dass Minijobber und Minijobberinnen im SGB-II-Bezug unabhängig davon, ob sie im rechtlichen Sinn als arbeitslos gelten - im Rahmen des „Aktivierungsregimes“ zu einer umfassenderen Erwerbsbeteiligung motiviert werden sollen, um die Hilfebedürftigkeit zu überwinden oder zumindest weiter zu reduzieren.

Vor diesem Hintergrund soll dann untersucht werden, ob die vom Gesetzgeber betonte Brückenfunktion der Minijobs für die Vermittlung in sozialversicherungspflichtige und Existenz sichernde Beschäftigung tatsächlich existiert oder sich eine Verstetigung der Kombination von ALG-II-Bezug und Minijob ergibt. Bei dauerhaften Kombinationen ist ferner zu überprüfen, ob diese aus Sicht der Leistungsbeziehenden „gewählt“ werden, weil die jeweilige Kombination von Transfers und geringfügiger Erwerbstätigkeit möglicherweise als „komfortable“ Situation betrachtet wird. Alternativ ist denkbar, dass vor allem diejenigen Leistungsempfänger in diesem Status unfreiwillig verharren, die nur geringe Chancen haben, eine höher entlohnte sozialversicherungspflichtige Beschäftigung zu finden. Darüber hinaus stellt sich die Frage, ob geringfügig Beschäftigte im SGB II gegebenenfalls diskriminiert werden, da sie im Vergleich zu anderen Minijobbern noch weniger Wahlmöglichkeiten haben und ggf. unter Androhung von Sanktionen in (schlecht bezahlte) Minijobs gedrängt werden können.

Um zu überprüfen, ob sich anhand dieser kurz skizzierten Szenarien eine spezifische Lebenssituation der geringfügig Beschäftigten im SGB-II-Bezug nachweisen lässt, wurden auf Basis der Daten des „Panels Arbeitsmarkt und soziale Sicherung“ (PASS) (vorgestellt im Abschnitt 3) der Umfang der Erwerbsbeteiligung der im Minijob Beschäftigten, insbesondere ihre Arbeitszeit und ihr Stundenverdienst, analysiert, wobei als Referenzgruppe immer wieder auf Minijobberinnen und Minijobber außerhalb des Leistungsbezugs rekurriert wurde (Abschnitt 4 und 5). Die für geringfügig Beschäftigte allgemein sowie für Leiharbeiter und Leiharbeiterinnen bereits formulierte These der "Lohndiskriminierung“ (Brehmer/Seifert 2008; Keller/Seifert 2009) wird konkretisiert. Um die Brückenfunktion der Minijobs zu überprüfen, werden Übergänge der eLb mit Minijob in sozialversicherungspflichtige Erwerbsarbeit untersucht und mit allen ALG-II-Beziehenden als Referenzgruppe verglichen (Abschnitt 6). Ob der Verbleib im ALG-II-Bezug plus Minijob auf „Freiwilligkeit“ oder „Alternativlosigkeit“ beruht, lässt sich nur indirekt beantworten, indem Deprivation, d.h. fehlende Versorgung mit materiellen (Konsum-)Gütern im Haushalt, Gesundheitszustand, subjektive Zufriedenheit und Haushaltseinkommen der verschiedenen Gruppen (ALG-II-Beziehende mit und ohne Minijob sowie Aussteiger) vergleichend analysiert werden (Abschnitt 7). Abschnitt 8 zieht ein Fazit.

\section{ALG-II-Beziehende mit Minijob - rechtliche Regelungen}

Durch Inkrafttreten des Zweiten Gesetzes für moderne Dienstleistungen am Arbeitsmarkt zum 1. April 2003 wurde die geringfügige Beschäftigung neu geregelt. Die Geringfügigkeitsgrenze wurde von $325 €$ auf $400 €$ monatlich angehoben, während die bisherige Zeitgrenze einer maximalen Wochenarbeitszeit von bis zu 15 Stunden aufgehoben wurde. Dies entsprach einer Rücknahme der Reform von 1999, die auf die Eindämmung geringfügiger Beschäftigung gerichtet war. Explizites beschäftigungspolitisches Ziel war es nunmehr, die geringfügige Beschäftigung zu fördern und auszuweiten.

ALG-II-Beziehende können ihr Haushaltseinkommen erhöhen, wenn sie Erwerbseinkommen beziehen. Zwar wird dieses Einkommen grundsätzlich mit dem ALG II verrechnet, ausgenommen ist jedoch ein Freibetrag von $100 €$. Verdienste über 100 und bis $800 €$ werden zu $80 \%$ mit den Leistungen nach SGB II verrechnet. Diese Anrechnungsregelung bedingt, dass erwerbsfähige Leistungsberechtigte mit einem Minijob von $400 €$ auf einen „Zuverdienst“ zur Grundsicherungsleistung von maximal $160 €$ kommen können (Dietz et al. 2011).

Aufgrund dieser Regelungen verfügen Personen, die ein Erwerbseinkommen erzielen, immer über ein Haushaltseinkommen, das höher ist als bei Personen, die ausschließlich Transferleistungen beziehen. Während die ersten $100 €^{3}$ des Erwerbseinkommens zu einem „sprunghaften“ Anstieg des Haushaltseinkommens führen, nimmt das verfügbare Einkommen danach sehr langsam zu (exakt um 20 Cent pro verdientem Euro). Das Verlassen des Leistungsbezugs respektive das Überwinden der Hilfebedürftigkeit variiert je nach Familiensituation. Für Alleinstehende wird diese Schwelle mit einem Bruttoeinkommen von ca. $1.200 €$ angegeben, bei Alleinerziehenden mit einem Kind ist der Ausstieg aus dem ALG-II-Bezug mit Förderung durch Wohngeld plus Kinderzuschlag bei etwa $1.250 €$ monatlichem Bruttoeinkommen erreicht (Bundesagentur für Arbeit 2008, S. $37 \mathrm{ff}$.$) .$

3 Dieser Freibetrag gilt für jedes Mitglied der Bedarfsgemeinschaft und dessen individuelles Einkommen. Verdiens te über 800 und bis $1.200 €(1.500 €$ bei Paaren mit Kindern oder Alleinerziehenden) wurden bis Sommer 2011 zu 90\% auf das Transfereinkommen angerechnet. Die seit der Reform des ALG II ab 1. Juli 2011 geltenden Freibeträge sehen eine Besserstellung von Einkommen im Bereich zwischen 800 und $1.000 €$ vor, wo nunmehr nur $80 \%$ angerechnet werden, womit der maximale Selbstbehalt von erwerbstätigen Leistungsberechtigten um $20 €$ steigt (Bundesministerium für Arbeit und Soziales 2010). Für den Bereich der geringfügigen Beschäftigung hat dies jedoch keine Auswirkungen. 


\section{Datenbasis: Panel Arbeitsmarkt und soziale Sicherung}

Die Datengrundlage der folgenden Analysen bildet, soweit nicht anders vermerkt, das „Panel Arbeitsmarkt und soziale Sicherung" (PASS), welches vom Institut für Arbeitsmarkt- und Berufsforschung (IAB) der Bundesagentur für Arbeit betreut wird. Die Panelbefragung zielt auf den unteren Einkommensbereich und die Haushalte mit ALG-IIBezug. Es wurden zwei Stichproben realisiert: Zum einen eine bevölkerungsrepräsentative Stichprobe mit einer Überrepräsentanz von ökonomisch eher schwächeren Wohngebieten, um ein ausreichend großes Sample an Haushalten mit niedrigen Einkommen zu generieren, damit detaillierte Analysen in diesem Einkommensbereich möglich und aussagekräftig werden. Zum anderen hat man ein Sample aus der ALG-II-Bezieher-Statistik gezogen. Damit sollen Analysen zum Verbleib und zum Ausstieg aus dem SGB-IILeistungsbezug möglich werden, die ansonsten aufgrund der geringen Fallzahl bei bevölkerungsweiten Stichproben häufig mit einer hohen Unsicherheit verbunden sind. Mit den ausgewählten Haushalten wurde ein Haushaltsinterview und mit allen Personen ab dem 16. Lebensjahr ein Personeninterview geführt (im Detail: Berg et al. 2010; Trappmann et al. 2010).

Diese Längsschnitt-Studie wird seit Ende 2006/1. Halbjahr 2007 jährlich durchgeführt. Derzeit stehen Daten aus drei Wellen bis einschließlich 1. Halbjahr 2009 zur Verfügung. Für die hier vorgestellte Datenanalyse wurde ein Längsschnittdesign gewählt: Basis bilden alle Personen zwischen 18 und 64 Jahren, mit denen in allen drei Erhebungen

ABB. 1

\section{Entwicklung der Anteile von Minijobs von 2007 bis 2009 bei ALG-II-Bezug und außerhalb des SGB II*}

Angaben in Prozent $\square$ ALG-II-Bezug $=$ kein ALG-II-Bezug $\square$ Gesamt

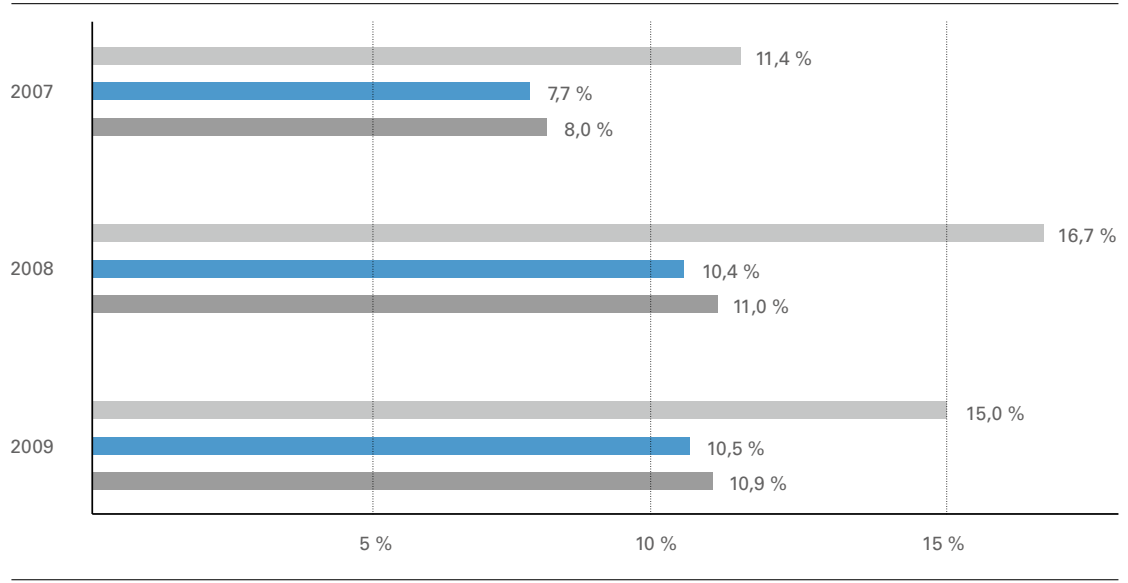

*Basis: Personen im Alter von 18 bis 64 Jahren.

Quelle: PASS Längsschnitt Welle 1-3, gewichtet, 18 bis 64-Jährige (in Welle 1). neben dem Haushaltsinterview auch ein Personeninterview geführt wurde und für die Informationen zum ALG-IIBezug und zur (Nicht-)Erwerbstätigkeit vorliegen. Durch entsprechende Gewichtungs- und Hochrechnungsfaktoren werden die durch dieses Design verursachten Verschiebungen weitgehend ausgeglichen.

\section{Verbreitung der Minijobs und sozio-demografische Merkmale der darin Beschäftigten}

Nach den Daten des PASS hat etwa jede zehnte Person zwischen 18 und 64 Jahren, die ALG II bezieht, mindestens einen Minijob (2007: 8\%; 2009: 10,9\%). In Westdeutschland lag der Anteil der ALG-II-Beziehenden mit einem Minijob immer etwas höher (2007: 12,1\%; 2009: 16,6\%) als in Ostdeutschland (10,7 \% bzw. 13,7\%). Der Anteil der geringfügig Beschäftigten mit ALG-II-Bezug an allen Personen mit Minijob liegt bei über $12 \%$, d. h. etwa jeder achte weilbliche oder männliche Minijobber bezieht gleichzeitig ALG II.

Die relative Bedeutung der Minijobs ist im ALG-II-Bezug höher als außerhalb des SGB II: Der Anteil der geringfügig Beschäftigten an den erwerbstätigen Leistungsbeziehern stieg von 11,4\% (Anfang 2007) auf $15 \%$ (2009). Der Anteil der ausschließlich geringfügig Beschäftigten außerhalb des ALG-II-Bezugs betrug dagegen 2009 nur 10,5\% (Abbildung 1).

Betrachtet man die sozio-demografischen Merkmale der geringfügig beschäftigten ALG-II-Beziehenden, so zeigen sich spezifische Gemeinsamkeiten und Unterschiede gegenüber den im Minijob Beschäftigten außerhalb des SGB II:

(1) Insgesamt üben Frauen im ALG-II-Bezug mit 18,4\% (2009) deutlich häufiger einen Minijob aus als Männer mit $11,2 \%$ im selben Jahr, wobei die Differenz zwischen den Geschlechtern im Zeitverlauf abnimmt. Die Dominanz der Frauen bei den Minijobs entspricht der Verteilung außerhalb des SGB-II-Bezugs. Hier sind jedoch die Quoten der Inanspruchnahme generell geringer (Frauen 16,1 \%, Männer 5,1\%) und die Geschlechterdifferenz ist größer.

(2) Der Anteil der Personen mit einem Minijob im ALGII-Bezug ist umso höher, je älter die Personen sind. So steigt der Anteil der geringfügig beschäftigten Erwerbstätigen 2009 von $4,8 \%$ bei den unter 25 -Jährigen bis auf $22,3 \%$ bei den über 57-Jährigen.

(3) Während außerhalb des ALG-II-Bezugs die Häufigkeit eines Minijobs mit der Höhe der Qualifikation abnimmt, gilt innerhalb des Leistungsbezugs, dass die Minijobberinnen und Minijobber in der Regel zumindest eine berufliche Ausbildung abgeschlossen haben. Für die Personen 
ALG-II-Bezug

\begin{tabular}{lccc}
\hline & Gesamt & Männer & Frauen \\
\hline Welle 1 (2007) & & & \\
\hline Verdienst & 250 & 140 & 250 \\
\hline Arbeitszeit & 10 & 12 & 10 \\
\hline Welle 3 (2009) & & & 165 \\
\hline Verdienst & 165 & 155 & 8 \\
\hline Arbeitszeit & 10 & 12 & \\
\hline
\end{tabular}

kein ALG-II-Bezug

\begin{tabular}{c|c|c}
\hline Gesamt & Männer & Frauen \\
\hline 318 & & \\
\hline 9 & 250 & 340 \\
\hline & 10 & 9 \\
\hline 300 & 200 & 300 \\
\hline 9 & 6 & 10 \\
\hline
\end{tabular}

Anmerkungen: Summe aller Minijobs; mittlerer Verdienst/mittlere Arbeitszeit = Median, d. h. Wert, unter bzw. über dem $50 \%$ der Befragten liegen.

Quelle: PASS Längsschnitt Welle 1 und 3, gewichtet, 18- bis 65-Jährige (in Welle 1).

im ALG-II-Bezug sind 2009 die Anteile der im Minijob Beschäftigten für die ungelernten Kräfte mit 10,2\% nur halb so hoch wie außerhalb des Leistungsbezugs, während bei den Personen mit beruflicher Ausbildung der Anteil mit 18,3\% um sieben Prozentpunkte höher liegt als bei Personen ohne ALG II und bei Personen mit mindestens einer Techniker- oder Meisterausbildung mit 14,9\% sogar doppelt so hoch ist.

\section{Entwicklung von Einkommen, Arbeitszeit und Stundenlöhnen}

Nachdem die quantitative Bedeutung der Minijobber im SGB II in den letzten Jahren deutlich zugenommen hat, werden hier ihre Einkommen und Stundenlöhne untersucht und es wird die These der „Lohndiskriminierung“ in Bezug auf die Minijobs für Personen im Leistungsbezug des ALG II überprüft. In einer Sonderauswertung hat die Bundesagentur für Arbeit für die Verdienste der ALG-II-Beziehenden für Dezember 2008 mehrere Schwerpunkte bei der Verteilung der monatlichen Einkommen identifiziert: Annähernd ein Drittel der erwerbsfähigen ALG-II-Empfänger nutzte nahezu ausschließlich die im Rahmen des Freibetrags definierte Einkommensgrenze, um das ALG II um bis zu $120 €$ aufzustocken. ${ }^{\circledR}$ Eine weitere Konzentration von $14 \%$ der erwerbstätigen Leistungsberechtigten mit Monatseinkommen zwischen 300 bis unter $400 €$ zeigt, dass eine Orientierung an den Freibetragsgrenzen kein durchgängiges Phänomen ist, sondern auch die Geringfügigkeitsgrenze einen deutlichen regulativen Einfluss ausübt.

Anhand der Analyse der PASS-Daten wurde für 2009 ein mittleres Monatseinkommen von geringfügig beschäftigten ALG-II-Beziehenden von $155 €$ für Männer und von $165 €$ für Frauen berechnet. Das Minijob-Einkommen von Personen außerhalb des Leistungsbezugs ist deutlich höher und lag 2009 für Männer bei $200 €$, während Frauen im Mittel sogar $300 €$ monatlich erzielten (Tabelle 1). Im Zeitverlauf zwischen 2007 und 2009 wird ein Rückgang der Höhe des mittleren Monatsverdienstes deutlich, was auf einen höheren Anteil von Personen mit einem niedrigeren Verdienst aus Minijobs hinweist. $^{\bullet}$

Während die Personen mit ALG-II-Bezug ein deutlich niedrigeres Minijob-Einkommen haben als die Personen außerhalb des Leistungsbezugs, zeigt sich bei der Wochenarbeitszeit das genaue Gegenteil: Trotz des deutlich niedrigeren mittleren Monatsverdienstes arbeiten Personen im ALG-II-Bezug im Rahmen des Minijobs im Mittel (Median) eher mehr Stunden pro Woche als Personen außerhalb des Leistungsbezugs. Dies kommt nicht zuletzt durch das vergleichsweise starke zeitliche Engagement der Männer im ALG-II-Bezug und deren vergleichsweise höherer Inanspruchnahme von Minijobs zustande, ${ }^{\oplus}$ während bei den Nicht-Leistungsbeziehenden Frauen genauso lange bzw. 2009 sogar etwas länger arbeiten als Männer (Tabelle 1).

Daraus lässt sich erstens schließen, dass Minijobs generell vielfach unterhalb der $400 €$ Grenze angeboten bzw. nachgefragt werden. Die häufig geäußerte Vermutung, dass ALG-II- Empfänger den Umfang ihres Arbeitsangebots primär an der Freibetragsgrenze orientieren und damit Einkommen und Zeiteinsatz „optimieren“, ist entsprechend zu relativieren. Zweitens implizieren die Daten zu monatlichem Verdienst und Arbeitszeiten, dass die geringfügig Beschäftigten im ALG-II-Bezug deutlich niedrigere Stundenlöhne erzielen als Minijobberinnen und Minijob-

$415,1 \%$ der erwerbstätigen Leistungsberechtigten erzielten bis zu $100 €$ und weitere $17,6 \%$ zwischen 100 und $200 €$.

5 Die Durchschnittsverdienste - hier nicht dargestellt - sind dabei tendenziell gleich geblieben.

(6 Bei Betrachtung der Durchschnittswerte fällt der Unterschied noch etwas höher aus. 
TABELLE 2

\section{Vergleich der Stundenlöhne im Minijob 2007 und 2009 (1. und 3. Welle)} Anteile in Prozent
Welle 1 (2007)

\begin{tabular}{lc|c}
\hline & ALG I & kein ALG II \\
\hline Unter $4 €$ & 24,2 & 10,1 \\
\hline $4 €$ bis unter $7 €$ & 47,6 & 31,9 \\
\hline $7 €$ bis unter $10 €$ & 22,1 & 41,3 \\
\hline $10 €$ und mehr & 6,2 & 16,8 \\
\hline Durchschnitt in Euro & $\mathbf{5 , 9 3} €$ & $\mathbf{7 , 8 4}$ \\
\hline
\end{tabular}

Anmerkungen: Stundenlöhne berechnet auf Basis der Arbeitszeit in Minijobs und des monatlchen Arbeitsverdienstes.

Quelle: PASS Längsschnitt Welle 1 und 3, gewichtet, 18- bis 65-Jährige.
Welle 3 (2009)

\begin{tabular}{c|c}
\hline ALG I & kein ALG II \\
\hline 35,1 & 16,6 \\
\hline 29,0 & 30,9 \\
\hline 22,8 & 29,1 \\
\hline 13,1 & 23,4 \\
\hline $\mathbf{6 , 0 8} €$ & $\mathbf{8 , 1 6} €$ \\
\hline
\end{tabular}

Mitteilungen

\section{ABB. 2}

\section{Stundenlöhne von Minijobs mit und ohne ALG II 2009}

Anteile in Prozent - $10 €$ und mehr $\square 7$ bis unter $10 € \square 4$ bis unter $7 € \quad$ unter $4 €$

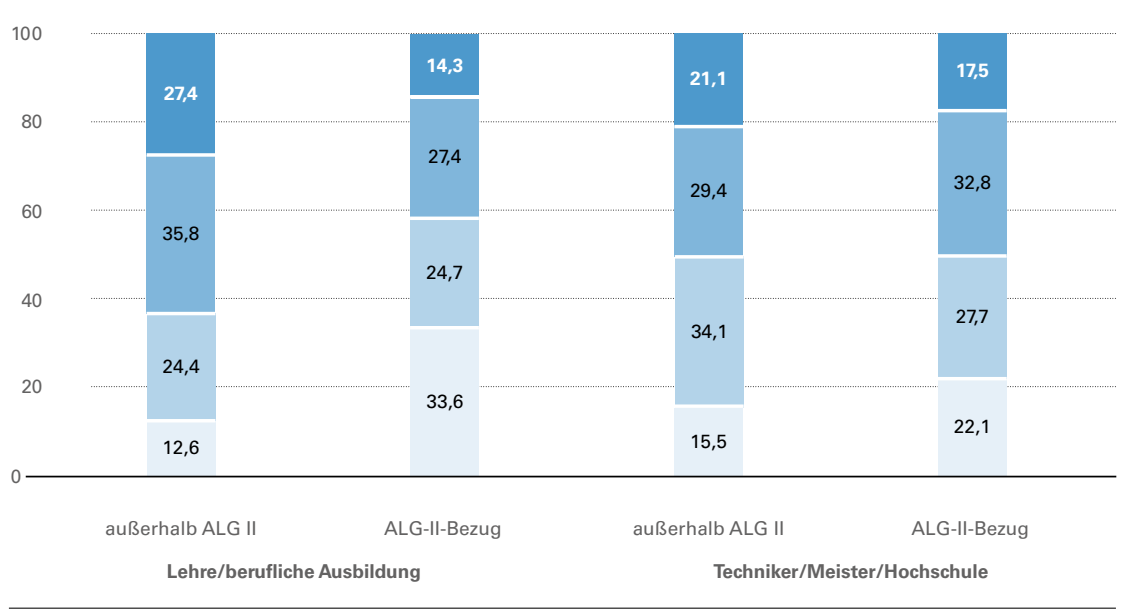

Anmerkung: Stundenlöhne berechnet auf Basis der Arbeitszeit in Minijobs und des monatlichen Arbeitsverdienstes.

Quelle: PASS Längsschnittwelle 1-3, gewichtet, 18 bis 64-Jährige (in Welle 1). die oberen und die unteren Segmente zugelegt haben. Mehr als ein Drittel der geringfügig Beschäftigten mit ALG II gibt 2009 an, dass sie insgesamt auf einen Stundenlohn von unter $4 €$ kommen. $13 \%$ kommen dagegen auf einen Stundenlohn von $10 €$ oder mehr.

Die entsprechenden Differenzen zwischen den Stundenlöhnen von Personen mit und ohne ALG II lassen sich jedoch nicht, wie man vielleicht vermuten könnte, durch Unterschiede im Qualifikationsniveau erklären. Auch wenn man nach Qualifikation kontrolliert, ist der Anteil derjenigen mit niedrigem Stundenlohn bei den Minijobbern im ALG-II-Bezug jeweils deutlich höher als bei Nicht-Leistungsbeziehenden (Abbildung 2).

Eine Regressionsanalyse, in der verschiedene Faktoren aufgenommen wurden, zeigt zwar, dass Ausbildungsniveau, Alter und Geschlecht einen signifikanten Einfluss auf die Höhe des Stundenlohnes haben. Darüber hinaus zeigt jedoch vor allem der Bezug bzw. Nichtbezug von ALG II weiterhin einen starken und signifikanten Einfluss auf die Höhe des Stundenlohnes. Den stärksten Einfluss hat jedoch die Region, sodass in Ostdeutschland auch die Kluft zwischen Leistungsbeziehern und nicht Leistungsbeziehern besonders stark ausgeprägt ist.

Es bleibt somit festzuhalten, dass die Einkommenschancen im Rahmen der Minijobs in Verbindung mit dem Leistungsbezug sowohl bezüglich der erzielten monatlichen Einkommen als auch der dabei erhaltenen Stundenlöhne deutlich schlechter sind als bei Minijobs, die von NichtLeistungsbeziehenden ausgeübt werden. Die These der Lohndiskriminierung bei atypisch Beschäftigten (vgl. dazu auch Voss/Weinkopf in diesem Heft) gilt damit in verstärktem Ausmaß für die Gruppe der Minijobber im ALG-IIBezug. Diese sind nicht nur gegenüber den erwerbstätigen Personen im Allgemeinen, sondern auch gegenüber den Minijobbern ohne ALG II benachteiligt. Der entsprechende Effekt zeigt sich verschärft für die entsprechende Gruppe in Ostdeutschland.

\section{Minijobber mit stabilem ALG-II-Bezug und Aussteiger
aus dem SGB II}

ber ohne Transferbezug. Dies lässt sich anhand des berech neten durchschnittlichen Stundenlohns bestätigen, der 2009 für geringfügig Beschäftigte im ALG-II-Bezug 6,08 € betrug, während er sich bei Personen ohne SGB-II-Leistungen auf immerhin $8,16 €$ belief (Tabelle 2).

Der Vergleich zwischen 2007 und 2009 zeigt, dass die Differenz zwischen den Gruppen weitgehend stabil blieb, sich aber eine leichte nominale Steigerung des mittleren Stundenlohns bei beiden Gruppen ergab. Diese geht jedoch vor allem bei den ALG-II-Beziehern mit einer stärkeren Spreizung der Stundenlöhne einher. Bei den Aufstockern hat die Konzentration auf das "mittlere“ Segment von $4 €$ bis unter $7 €$ Stundenlohn deutlich abgenommen, während
Als nächstes soll untersucht werden, ob die Minijobs die ihnen zugedachte Brückenfunktion tatsächlich erfüllen oder sich eher dauerhafte Kombinationen von Minijob und Transferbezug im Rahmen des SGB II herausbildet haben. Bei der Auswertung der PASS-Daten wurde ein „stabiler“ Leistungsbezug bzw. Minijob dann identifiziert, wenn zu Merkmale vorlagen. ${ }^{\circ}$ Die Mehrzahl der Personen (68\%), die in der ersten Welle ALG II bezogen, verbleibt auch in den beiden Folgejahren im Leistungsbezug. Nur 29,1 \% der ALG-II-Beziehenden der ersten Welle konnten den Leisden jeweiligen Befragungszeitpunkten jeweils diese beiden 
tungsbezug im Verlauf der nächsten beiden Jahre verlassen und erhielten zum Zeitpunkt der dritten Erhebung 2009 keine SGB-II-Leistungen mehr.

Inwiefern die Stabilität des Minijobs mit der Stabilität des Bezugs von ALG II korrespondiert, lässt sich nach dem Verlaufstyp des ALG-II-Bezugs klären. Stabile Bezieherinnen und Bezieher von ALG II üben zu etwas mehr als einem Viertel in allen drei Jahren einen Minijob aus, während annähernd ein Drittel von ihnen zu keinem Befragungszeitpunkt geringfügig beschäftigt war. Umgekehrt haben von den Personen, die den ALG-II-Bezug verlassen haben, mehr als die Hälfte zu keinem der drei Erhebungszeitpunkte einen Minijob ausgeübt. Zum Zeitpunkt der letzten Befragung vor dem Ausstieg aus dem SGB II waren $15,5 \%$ in einem Minijob tätig ( $5 \%$ in jedem Jahr, 4,4\% nach einer Unterbrechung und 6,1\% haben den Minijob erst nach oder zeitgleich mit ihrem Ausstieg aufgenommen (Abbildung 3). ${ }^{\mathbf{0}}$

Der Vergleich dieser Verlaufstypen zeigt, dass ein Ausstieg aus dem ALG II tendenziell wahrscheinlicher ist, wenn vorher kein Minijob ausgeübt wurde. Entsprechend scheinen Minijobs im SGB II häufig von jenen Personen ausgeübt zu werden, deren Ausstiegschancen aus dem Transferbezug aus welchen Gründen auch immer - gering sind. Für einen (kleineren) Teil der geringfügig Beschäftigten im SGB II scheinen Minijobs jedoch auch im Sinne einer Art Brücke in reguläre Beschäftigung zu wirken. So sind $95 \%$ derjenigen, die im Leistungsbezug einen Minijob ausübten, nach dem Verlassen des SGB II oberhalb der Minijobschwelle erwerbstätig. Rund drei Viertel von ihnen arbeitet Vollzeit, weitere $14 \%$ Teilzeit zwischen 21 und 35 Stunden. Lediglich $8 \%$ arbeiten zwischen zwölf und unter 21 Stunden und nur $3 \%$ haben noch kürzere Arbeitszeiten. Allerdings ist dabei zu berücksichtigen, dass mehr als ein Viertel der Aussteiger in Erwerbstätigkeit zunächst in eine befristete Tätigkeit übergeht (29\%). Wie stabil deren Erwerbstätigkeit über die Zeit hinweg ist, lässt sich mit den hier vorgestellten Analysen nicht beurteilen. Jüngere Untersuchungen von Koller und Rudolph (2011) weisen jedoch darauf hin, dass ein hoher Anteil der Beschäftigungsverhältnisse von Aussteigerinnen und Aussteigern aus dem SGB II nicht von Dauer ist. Nach ihren Zahlen hat nur etwas mehr als die Hälfte von ihnen länger als sechs Monate ein Beschäftigungsverhältnis, das ohne zusätzliche Transferbezüge als Existenz sichernd gilt.

Die Daten bestätigen, dass Minijobs in ihrer Gesamtheit die Überwindung des Leistungsbezugs nicht maßgeblich befördern. Für eine kleine Gruppe können die unterhalb der Sozialversicherungspflicht angesiedelten Erwerbstätigkeiten jedoch durchaus dazu beitragen, den Übergang aus der Hilfebedürftigkeit zu erleichtern. Eine generelle Brückenfunktion aus dem ALG-II-Bezug kommt den Minijobs damit nicht zu. Vielmehr spricht vieles dafür, dass die geringfügige Tätigkeit zur Verbesserung der finanziellen Situation genutzt wird, weil kurz- oder langfristig keine andere Option zur Verfügung steht bzw. kein Ausstieg aus dem Leistungsbezug erwartet werden kann. ${ }^{\ominus}$
ABB. 3

\section{Minijobber im SGB II nach Stabilität des Leistungsbezugs}

Angaben in Prozent

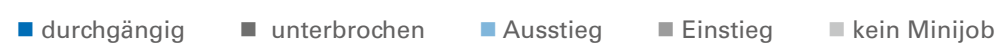
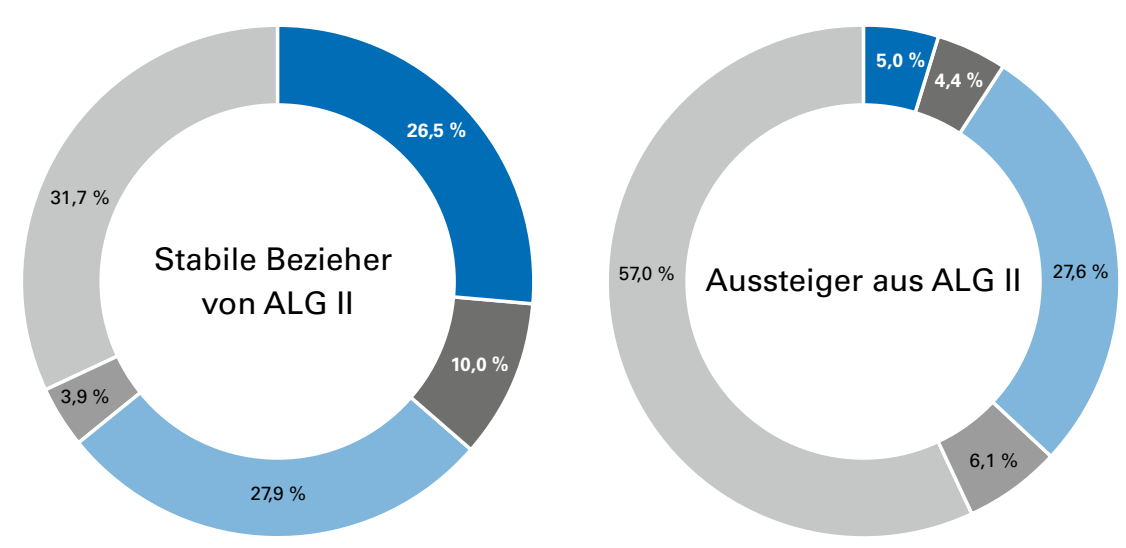

Quelle: PASS Längsschnitt Welle 1-3, gewichtet, 18 bis 64-Jährige (in Welle 1).

\section{Zufriedenheit und gesellschaftliche Teilhabe}

Abschließend soll die Frage aufgeworfen werden, ob es Hinweise darauf gibt, dass der Verbleib im ALG-II-Bezug in Kombination mit einem Minijob auf „Freiwilligkeit“ oder „Alternativlosigkeit“ beruht. Zur Annäherung an eine Antwort werden die Zufriedenheit und die gesellschaftliche Teilhabe von Minijobbern im Transferbezug des SGB II untersucht.

Vergleicht man erwerbstätige Leistungsberechtigte im SGB II mit solchen Personen, die den ALG-II-Bezug verlassen haben, weisen die subjektiven Indikatoren und das Haushaltseinkommen in die zu erwartende Richtung: Die Lebenszufriedenheit und das Haushaltseinkommen

(7) Unterbrechungen, die zwischen die jährlichen Erhebungen fallen, bleiben bei der Messung der Stabilität des Leistungsbezugs folglich unberücksichtigt. Aufgrund dieses methodischen Vorgehens fallen die Stabilitätsquoten höher aus als bei der Zugrundelegung von Prozessdaten der BA.

8 Lediglich bei dieser Gruppe kann man vermuten, dass bei einem Teil von ihnen der Ausstieg durch die Aufnahme eines Minijobs erfolgte.

(9 Der Beitrag von Klenner und Schmidt in diesem Heft zeigt für weibliche Erwerbsverläufe u.a., wie entscheidend in Bezug auf Aspekte wie Armuts- und Prekaritätsrisiken die Dauer des Zeitraumes ist, in dem Minijobs ausgeübt worden sind. 
TABELLE 3

\section{Deprivation, Haushaltseinkommen, Beurteilung des Lebensstandards und Zufriedenheit}

Durchschnittswerte

\begin{tabular}{|c|c|c|c|c|c|c|c|c|}
\hline & \multicolumn{4}{|c|}{$\begin{array}{c}\text { Bezieher von ALG II } \\
\text { Minijob }\end{array}$} & \multicolumn{4}{|c|}{$\begin{array}{c}\text { Aussteiger aus ALG II } \\
\text { Mini Job }\end{array}$} \\
\hline & Gesamt & Durchgängig & Beendet & Kein & Gesamt & Durchgängig & Beendet & Kein \\
\hline Deprivation ${ }^{11}$ & 6,9 & 8,2 & 6,7 & 5,7 & 4,5 & 5,2 & 4,5 & 4,8 \\
\hline Akt. Lebensbedingungen ${ }^{21}$ & 4,6 & 4,7 & 5,0 & 5,1 & 5,9 & 5,5 & 5,6 & 5,4 \\
\hline Zuk. Lebensbedingungen ${ }^{21}$ & 5,0 & 4,5 & 5,6 & 5,5 & 6,2 & 5,4 & 6,7 & 5,8 \\
\hline Gesundheit $^{31}$ & 6,0 & 5,3 & 6,3 & 5,6 & 7,0 & 5,5 & 6,5 & 7,1 \\
\hline Lebensstandard $^{31}$ & 5,2 & 4,6 & 5,2 & 5,7 & 6,3 & 5,9 & 6,0 & 6,1 \\
\hline Generelle Lebenszufriedenheit ${ }^{31}$ & 5,8 & 5,7 & 5,9 & 6,3 & 6,6 & 7,4 & 6,4 & 6,4 \\
\hline Soziale Teilhabe ${ }^{4)}$ & 6,2 & 5,8 & 5,9 & 7,1 & 7,1 & 7,9 & 8,2 & 6,9 \\
\hline Gew. Haushaltseinkommen ${ }^{5 !}$ & $705 €$ & $730 €$ & $742 €$ & $865 €$ & $957 €$ & $978 €$ & $978 €$ & $935 €$ \\
\hline \multicolumn{9}{|c|}{$\begin{array}{l}\text { 1) Haushaltsfragebogen - ungewichteter Index basierend auf } 26 \text { Items: Das Item wird gezählt, wenn es aufgrund fin } \\
\text { Skala von } 0=\text { kein Item fehlt aufgrund finanzieller Gründe bis } 26-\text { alle } 26 \text { Items fehlen aufgrund finanzieller Grür } \\
\text { 2) Haushaltsfragebogen: Die } 11 \mathrm{er}-\text { Skala geht von } 0=\text { sehr schlecht bis } 10=\text { sehr gut; } \\
\text { 3) Personenfragebogen: Die } 11 \mathrm{er}-S k a l a \text { geht von } 0=\text { ganz und gar unzufrieden bis } 10=\text { ganz und gar zufrieden; } \\
\text { 4) Personenfragebogen: Die } 10 \mathrm{er}-S k a l a \text { geht von } 1=\text { Ausgeschlossen bis } 10=\text { Dazugehörig; } \\
\text { 5) Haushaltsnettoäquivalenzeinkommen: mittels der neuen OECD-Skala angepasstes Haushaltsnettoeinkommen. }\end{array}$} \\
\hline \multicolumn{8}{|c|}{ Quelle: PASS Längsschnitt Welle 1-3, gewichtet, 18- bis 64-Jährige. } & NSI Mitteilungen \\
\hline
\end{tabular}

sowie die soziale Teilhabe ist dann höher, wenn man nicht mehr auf die Transferleistung angewiesen ist. Die Deprivation, d. h. die aufgrund der finanziellen Situation fehlende Versorgung mit materiellen (Konsum-)Gütern im Haushalt, ist entsprechend geringer, wenn man keine ALG-II-Leistungen mehr bezieht (Tabelle 3).

Für die „dauerhaften“ Bezieherinnen und Bezieher von ALG II zeigte die Ausübung eines Minijobs zwischen 2007 und 2009, dass die Gruppe der durchgängigen Aufstocker (alle drei Zeitpunkte der Erhebung) im ALG II mit Minijob die höchsten Werte bei der Deprivation sowie eine klar geringere Lebenszufriedenheit, soziale Teilhabe und stärker negative Zukunftserwartungen, einschließlich einer schlechteren Einschätzung der eigenen Gesundheit hat und zwar auch im Vergleich zu denjenigen ALG-II-Beziehenden, die nie einen Minijob ausübten (Tabelle 3). Trotz der Erzielung von Erwerbseinkommen durch den Minijob wird der Lebensstandard im Vergleich zu anderen ALG-IIBeziehenden deutlich schlechter bewertet. Eine Erklärung dafür liegt sicher darin, dass das äquivalenzgewichtete Haushaltseinkommen der Minijobber unterdurchschnittlich ist, was teilweise auf die Zusammensetzung der jeweiligen Bedarfsgemeinschaften zurückzuführen sein dürfte.

Die langanhaltende bzw. dauerhafte Exklusion von sozialversicherungspflichtiger Beschäftigung und die fehlende Chance zu einer maßgeblichen Verbesserung der Einkommenssituation münden insbesondere für Personen, die dauerhaft einen Minijob ausüben, offenbar in eine erhöhte Frustration. Bezieherinnen und Bezieher von ALG II, die ihren Minijob beendet haben, beurteilen ihre konkrete Lebenssituation hingegen etwas positiver und erwarten auch für die nahe Zukunft eine etwas bessere Entwicklung. Be- trachtet man die Aussteiger aus dem ALG-II-Bezug, so gilt auch hier die Regel, dass die noch in Minijobs Beschäftigten hinsichtlich der subjektiven Indikatoren und des Haushaltseinkommens ebenfalls etwas schlechter abschneiden als Personen, die ihren Minijob beendet haben bzw. im Untersuchungszeitraum keinen Minijob ausgeübt haben. ${ }^{(0)}$

Das aber verdeutlicht: Minijobs in Kombination mit ALG-II-Bezug führen trotz Verbesserung der finanziellen Situation des Haushalts nicht durchgängig zu höherer $\mathrm{Zu}$ friedenheit und sozialer Teilhabe. Ein (dauerhaft ausgeübter) Minijob legt nahe, dass es individuelle wie strukturelle Probleme gibt, die es der Person erschweren, den ALG-IIBezug zu überwinden. Die Unzufriedenheit mit der Gesundheit v. a. bei den dauerhaft in Minijobs Beschäftigten lässt vermuten, dass hier anhaltende Restriktionen bestehen, den Status der Integration in den Arbeitsmarkt zu verbessern. Das vergleichsweise niedrige Äquivalenzeinkommen kann als Hinweis auf größere Bedarfsgemeinschaften interpretiert werden, für die es auch aus diesem Grund schwerer ist, den ALG-II-Bezug zu überwinden. Darüber hinaus ist denkbar, dass auch eine Gruppe der aktiveren und stärker erwerbsorientierten ALG-II-Beziehenden geringfügig beschäftigt ist, gleichzeitig aber ihre Lebenssituation aufgrund des Verbleibs im Leistungsbezug auch kritischer beurteilt als andere. Es kann demnach vermutet werden, dass sich die Motive zur Aufnahme bzw. Beibehaltung eines Minijobs bei den ALG-II-Beziehenden ebenso stark unterscheiden wie die Chancen auf "gute“ oder „schlechte“ Minijobs.

(1) Eine Ausnahme bildet dabei die generelle Lebenszufriedenheit. 


\section{Schlussfolgerungen}

Die vorangegangene Analyse hat in Bezug auf die Lebensund Arbeitsmarktsituation der Gruppe der ALG-II-Beziehenden mit Minijob - im Vergleich zu den verschiedenen Referenzgruppen - einige interessante Erkenntnisse erbracht. Die von den erwerbstätigen Leistungsberechtigten in den Minijobs erzielten Stundenlöhne sind erheblich niedriger als die von Minijobbern außerhalb des SGB-IILeistungsbezugs. ALG-II-Beziehende sind somit in besonderer Weise von Lohndiskriminierung betroffen. Da die niedrigeren Stundenlöhne der geringfügig erwerbstätigen Leistungsberechtigten gerade nicht durch eine niedrigere formale Qualifikation erklärt werden können, dürfte sich darin die Funktion des ALG II als allgemein verfügbare Lohnsubvention widerspiegeln. Es ist nicht unwahrscheinlich, dass die Vertragsparteien den Grundsicherungsbetrag in das Minijob-Vertragsverhältnis „hineinrechnen“, sodass faktisch „ein Kombilohn zu Lasten des Fiskus“ (Deutscher Frauenrat 2010, S. 5) entsteht, die Wirtschaft folglich Lasten auf die Allgemeinheit abwälzt. ${ }^{\text {(1) }}$

Diese Praxis wird letztlich gestützt durch das „Aktivierungsregime“ im SGB II, das einen eigenständigen Druck auf die Leistungsbeziehenden ausübt, Erwerbstätigkeiten auch im Niedrig(st)lohnbereich aufzunehmen. Zwar hat die Bundesagentur für Arbeit nach einem Gerichtsurteil in Stralsund, wo ein Arbeitgeber einen Stundenlohn von 1,32€ zahlte, einen Leitfaden "Lohnwucher" ${ }^{\text {(1) }}$ herausgegeben, in dem die Jobcenter aufgefordert werden, Fälle von Aufstockern zu prüfen, „in denen Kunden ein auffällig geringes Arbeitsentgelt erzielen und deshalb ergänzend auf den Bezug von SGB-II-Leistungen angewiesen sind“. Allerdings liegt die in diesem Zusammenhang genannte Entgelthöhe bei $3 €$ pro Stunde (Süddeutsche Zeitung vom 4.3.2010) und bietet den ALG-II-Beziehenden somit keinen Schutz vor dem Zwang zur Aufnahme von Niedriglohntätigkeiten. Vor allem die am Arbeitsmarkt tendenziell Chancenlosen unterliegen auch weiterhin dem Risiko der Lohndiskriminierung in verstärktem Maße.

Gleichwohl versuchen ALG-II-Beziehende, durch eine geringfügige Beschäftigung einen Zugang zum Arbeitsmarkt zu finden und die finanzielle Situation ihres Haushalts zu verbessern. Die Aufnahme eines Minijobs ist eher eine Reaktion „aus der Not“ als eine „Optimierung“ von Zeit und Geld, ${ }^{\circledR}$ wie nicht zuletzt aus den subjektiven Bewertungen der Teilhabe ersichtlich ist. Insbesondere die Gruppe der ALG-II-Beziehenden mit durchgängiger geringfügiger Beschäftigung fühlt sich daher stark depriviert und von gesellschaftlicher Teilhabe ausgeschlossen.

Ferner wurde deutlich, dass die im Zeitverlauf wachsende Zahl von ALG-II-Beziehenden, die den Leistungsbezug mit Einkommen aus geringfügiger Beschäftigung kombiniert, die Ausstiegschancen aus dem Leistungsbezug im Vergleich zu Nichtbeschäftigten mittelfristig nicht deutlich verbessern kann. Eine allgemeine Brückenfunktion der Minijobs in den regulären Arbeitsmarkt ist nicht auszumachen bzw. bleibt bestenfalls auf eine sehr kleine Gruppe beschränkt.

Wenngleich die Analyse quantitativer Daten eine umfassende Interpretation nicht erlaubt, legen die oben dargestellten Untersuchungsergebnisse nahe, dass für eine spezifische Gruppe von Leistungsbeziehenden die Ausübung eines Minijobs die einzige residuale Form der Arbeitsmarktpartizipation darstellt, da sie letztlich von sozialversicherungspflichtiger und Existenz sichernder Beschäftigung dauerhaft ausgeschlossen ist. Dies wurde auch durch qualitative Untersuchungen bestätigt (vgl. Dingeldey/Jäger 2010).

Bei den politischen Reformvorschlägen wäre entsprechend zu berücksichtigen, dass vielfach individuelle Eigenschaften der entsprechenden Leistungsbeziehenden den Übergang in reguläre Beschäftigung behindern, sodass für diese Gruppe letztlich nur spezifische Fördermaßnahmen zur Aufhebung des permanenten Leistungsbezuges und der damit einhergehenden sozialen Ausgrenzung beitragen können. Die immer wieder diskutierten Ausweitungen oder Einschränkungen der Freibetragsgrenzen (Bruckmeier et al. 2010; Dietz et al. 2011) gehen insofern am eigentlichen Problem vorbei, als das Ausmaß der geringfügigen Beschäftigungen bei den Aufstockern weniger durch die „Anreize“ für Arbeitsuchende als vielmehr durch die typischen Strukturen der entsprechenden Arbeitsmärkte (Voss/Weinkopf in diesem Heft) beeinflusst wird. Diese sind ihrerseits stark durch das Fehlen eines gesetzlichen Mindestlohnes einerseits und die Verfügbarkeit einer allgemeinen Lohnsubvention in Form des ALG II und der durch Schutzlosigkeit der ALG-II-Beziehenden gegen „Vermittlung“ in niedrig(st) entlohnte Tätigkeiten andererseits geprägt.

Zur Förderung der Übergänge in sozialversicherungspflichtige Beschäftigung müssten folglich weniger die finanziellen Anreize für die Leistungsempfängerinnen und Leistungsempfänger verändert werden. Es müsste vielmehr der Übergang von geringfügiger zur sozialversicherungspflichtigen Beschäftigung noch "weicher" gestaltet werden als bislang. Das beinhaltet auch, spezifische Arbeitsmärkte, die primär durch geringfügige Beschäftigung dominiert sind, stärker zu limitieren. Weitere politisch relevante Stellschrauben sind die Festlegung einer Untergrenze für die (Stunden-)Löhne (Mindestlohn) und die Einführung von „Zumutbarkeits“-Kriterien für die Arbeitsaufnahme der Transferbeziehenden im SGB II, die sich an gesellschaftlich erwünschten Zielen orientieren.

(1) Siehe das beschriebene Beispiel des Unternehmens KIK im Beitrag von Hinz in diesem Heft.

(12) Dieser ist Bestandteil der Fachlichen Hinweise der BA zu § 33 SGB II.

(3) So gibt es beispielsweise keine Belege dafür, dass Mehrfach-Minijob-Beschäftigungen in Bedarfsgemeinschaften massiv genutzt werden, um die Freibetragsregelung voll auszuschöpfen. 


\section{LITERATUR}

Berg, M./Cramer, R./Dickmann, C./Gilberg, R./Jesske, B./Marwinski, K./ Gebhardt, D./Wenzig, C./Wetzel, M. (2010): Codebuch und Dokumentation des Panel Arbeitsmarkt und soziale Sicherung (PASS), Welle 3 (2008/2009), Forschungszentrum der BA im IAB, in: FDZ Datenreport (06), Nürnberg, S. 197 Brehmer, W./Seifert, H. (2008): Sind atypische Beschäftigungsverhältnisse prekär? Eine empirische Analyse sozialer Risiken, in: Zeitschrift für ArbeitsmarktForschung 41 (4), S. 501-531

Brenke, K. (2008): Hilfebedürftig trotz Arbeit? - kein Massenphänomen in Deutschland, in: DIW Wochenbericht 75 (04), S. 33-41

Bruckmeier, K./Feil, M./Walwei, U./Wiemers, J. (2010): Reform der Hinzuverdienstmöglichkeiten im SGB II: Was am Ende übrig bleibt, IAB Kurzbericht (24), Nürnberg

Bruckmeier, K./Graf, T./Rudolph, H. (2007): Aufstocker - bedürftig trotz Arbeit, in: IAB - Kurzbericht (22), S. 1-8

Bruckmeier, K./Graf, T./Rudolph, H. (2008): Working poor: Arm oder bedürftig? in: IAB - Discussion Paper (34), S. 1-32

Bundesagentur für Arbeit (2008): Grundsicherung für Arbeitsuchende: Bedarfe, Leistungen und Haushaltsbudget, Bericht der Statistik der BA, Nürnberg

Bundesagentur für Arbeit (2009): Erwerbstätigkeit von erwerbsfähigen Leistungsbeziehern, September, Nürnberg

Bundesagentur für Arbeit (2010a): Grundsicherung für Arbeitsuchende:

Erwerbstätige Arbeitslosengeld II-Bezieher: Begriff, Messung, Struktur und

Entwicklung, Bericht der Statistik der BA, Nürnberg

Bundesagentur für Arbeit (2010b): Statistik nach Themen. Grundsicherung für Arbeitsuchende, Erwerbstätige Arbeitslosengeldbezieher, Nürnberg Bundesagentur für Arbeit (2011a): Analyse der Grundsicherung für Arbeitsuchende, August, Nürnberg

Bundesagentur für Arbeit (2011b): Der Arbeits- und Ausbildungsmarkt in Deutschland, Monatsbericht März, Nürnberg

Bundesministerium für Arbeit und Soziales (2010): Pressemitteilung: Gezielt Bildungsleistungen sind ein Riesenfortschritt für die Kinder, Bundeskabinet beschließt Gesetzentwurf zur SGB II-Leistungsreform, 16.11., Berlin

Deutscher Frauenrat (2010): Minijobs - Wege in die Armut. Kann sich unsere Gesellschaft Minijobs leisten? Positionspapier des Deutschen Frauenrates zu Minijobs, Berlin

Dietz, M./Koch, S./Rudolph, H./Walwei, U./Wiemers, J. (2011): Reform der Hinzuverdienstregeln im SGB II, in: Sozialer Fortschritt 60 (1/2), S. 4-15 Dingeldey, I. (2002): Das deutsche System der Ehegattenbesteuerung im europäischen Vergleich, in: WSI-Mitteilungen 55 (3), S. 154-161

Dingeldey, I./Jäger, C. (2010): „Wenn ich das nicht hätte, hätte ich gar nichts”. Zur Motivlage von ALG II Aufstockern mit Minijob. Abschlussbericht des Projektes Governance des Einkommensmix. Geringfügige Beschäftigung plus ALG II-Bezug, Institut Arbeit und Wirtschaft, Bremen

Greve, G./Pfeiffer, R./Vennebusch, T. (2007): Minijob-Zentrale - Entwicklung und Status quo der geringfügigen Beschäftigung, in: RV aktuell, Amtliches Mitteilungsblatt der Deutschen Rentenversicherung (54), S. 38-43

Institut Arbeit und Qualifikation (IAQ)/Institut Arbeit und Wirtschaft (FIA)/ Forschungs- und Kooperationsstelle Arbeit, Demokratie, Geschlecht (GendA) (2009): Bewertung des SGB II aus gleichstellungspolitischer Sicht, Abschlussbericht, Duisburg et al.
Keller, B./Seifert, H. (2009): Atypische Beschäftigungsverhältnisse: Formen, Verbreitung, soziale Folgen, in: Aus Politik und Zeitgeschichte (27), S. 40-46 Kirsch, J./Klein, M./Lehndorff, S./Voss-Dahm, D. (1999): Darf's etwas weniger sein? Arbeitszeiten und Beschäftigungsbedingungen im Lebensmitteleinzelhandel. Ein europäischer Vergleich, Berlin

Koch, A./Bäcker, G. (2004): Mini- und Midi-Jobs - Frauenerwerbstätigkeit und Niedrigeinkommensstrategien in der Arbeitsmarktpolitik, in: Baatz, D./Rudolph C./Satilmis, A. (Hrsg): Hauptsache Arbeit. Feministische Perspektiven auf den Wandel von Arbeit, Münster, S. 85-103

Koller, L./Rudolph, H. (2011): Viele Jobs von kurzer Dauer, IAB-Kurzbericht (14), Nürnberg

Die Minijobzentrale (2011): Geringfügige Beschäftigung. Knappschaft-BahnSee, http://www.minijob-zentrale.de/nn_10774/DE/2_AG/1_geringfuegige_ beschaeftigung/5_pauschalabgaben/pauschalabgaben.html (letzter Zugriff: 10.2.2011)

Rheinisch-Westfälisches Wirtschaftsinstitut (2004): Aspekte der Entwicklung von Mini-Jobs, Gutachten im Auftrag der Minijob-Zentrale der Bundesknappschaft, Endbericht - Zusammenfassung, Essen

Trappmann, M./Gundert, S./Wenzig, C./Gebhardt, D. (2010): PASS: a household panel survey for research on unemployment and poverty, in: Schmollers Jahrbuch, Zeitschrift für Wirtschafts- und Sozialwissenschaften 130 (4), S. 609-622

Winkel, R. (2005): Minijob-Bilanz: Kaum Lohnfortzahlung bei Krankheit und Mutterschaft. Lohnausgleichskasse machte 136,6 Millionen Überschuss, in: Soziale Sicherheit 54 (9), S. 292-298

\section{AUTOREN}

IRENE DINGELDEY, Dr., ist Forschungsleiterin der Abteilung Wandel der Arbeitsgesellschaft am Institut Arbeit und Wirtschaft (IAW) der Universität Bremen. Arbeitsschwerpunkte: Arbeitsmarkt-, Familien- und Lohnpolitik unter besonderer Berücksichtigung der Gender Aspekte sowie der Governance des Wohlfahrtsstaates.

dingeldey@iaw.uni-bremen.de

PETER SOPP, Dr., ist Mitarbeiter im Forschungsteam Internationaler Arbeitsmark (FIA), Berlin; PMS Markt- und Sozialforschung München. Arbeitsschwerpunkte: Arbeitsmarktforschung, Markt- und Sozialforschung.

peter.sopp@fia-institut.de

ALEXANDRA WAGNER, Dr., ist Geschäftsführerin des Forschungsteams Internatio naler Arbeitsmarkt (FIA), Berlin. Arbeitsschwerpunkte: Arbeitsmarkt- und Beschäftigungspolitik, Führungskräfte, Gender.

wagner@fia-institut.de 